\title{
Pushing the boundaries of total scattering methods
}

ISSN 2052-2525

NEUTRON|SYNCHROTRON

Keywords: commentary; grazing incidence; pair distribution function; thin films.

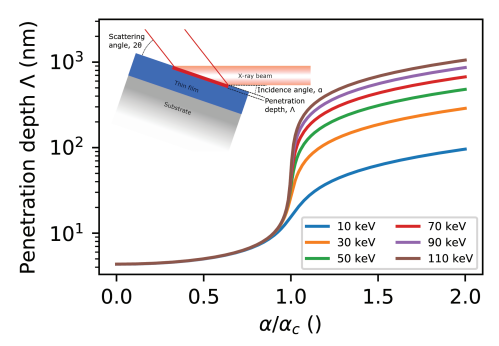

OPEN $\odot$ ACCESS
Robert J. Koch*

Condensed Matter Physics and Materials Science, Brookhaven National Laboratory, Upton, NY 11973, USA. *Correspondence e-mail: rkoch@bnl.gov

Total scattering and atomic pair distribution function (PDF) analysis, an extension of powder diffraction, has proven exceptionally useful for probing both local and long-range structure across a wide variety of materials (Billinge \& Kanatzidis, 2004; Egami \& Billinge, 2012; Keen \& Goodwin, 2015). The application of PDF analysis was initially limited to materials completely lacking long-range order (Guinier, 1994; Warren, 1969). Over the last two decades, developments in instrumentation (Chupas et al., 2003) and software (Proffen \& Billinge, 1999; Proffen \& Neder, 1999; Peterson et al., 2000; Billinge et al., 2002; Qiu et al., 2004; Tucker et al., 2007; Juhás et al., 2013) have allowed scientists to leverage computational advances and bring PDF to the forefront of structural characterization, extending its applicability well beyond amorphous materials. In this issue of IUCrJ, Dippel et al. (2019) report a new method for carrying out grazing-incidence PDF (GIPDF) measurements. This optimized GIPDF configuration has the potential to broaden the horizons of the total scattering field even further.

The PDF $G(r)$ is obtained through a through a Fourier transform of the normalized diffraction intensity $I(Q)$ measured up to a momentum transfer $Q$ typically greater than $20 \AA^{-1}$. While $G(r)$ and $I(Q)$ contain identical information, the PDF provides a unique 'real-space' view, giving the likelihood of finding pairs of atoms at a certain distance $r$. As assumptions regarding periodicity are not necessary, PDF analysis has proven particularly useful for nanomaterials (Masadeh et al., 2007; Page et al., 2011), materials with poor or only anisotropic crystallinity (Vogt et al., 2002; Gao et al., 2017) and crystalline systems with local structural distortions (Billinge et al., 1996; Senn et al., 2016).

Total scattering techniques place Bragg and diffuse scattering on equal footing, and thus it is ideal if the signal represents only the material of interest. This necessitates careful measurement and subtraction of the scattering signal from non-sample (background) materials in the beam path. For bulk powders measured in the standard transmission geometry at modern synchrotrons, this is usually straightforward, as the signal-tobackground ratio is large.

In the case of thin films on thick substrates, background subtraction in a transmission geometry is critically important and at times a limitation. As film thicknesses decreases relative to the substrate, the contribution of the film to the scattered signal similarly decreases. The result is often that the measured signal is dominated by the substrate, while the signal from the film of interest is buried in noise. While this issue can be remedied by increasing measurement times compared with those for standard bulk powders, the necessary times are often unfeasible. This has limited the applicability of PDF analysis to thin films, imposing hard restrictions on both film thickness and temporal resolution.

The recent work of Dippel et al. (2019) represents a significant advancement in the field of X-ray total scattering and PDF analysis. They exploit the grazing-incidence geometry shown inset in Fig. 1, where the X-ray beam meets the sample at an angle below that of total external reflection. In such a configuration, under ideal conditions the penetration depth of X-rays is independent of wavelength, and typically less than a nanometre, as shown in Fig. 1. This provides enhanced surface sensitivity and a significantly improved signal-to-background ratio, driving down the thickness detection limit.

Their work makes several key improvements on past GIPDF geometries. By using hard X-rays combined with a fast two-dimensional detector they achieve PDF resolutions and count times comparable with what is seen in more standard transmission geometries for bulk powders. Additionally, careful control over the beam footprint yields manageable instrumental resolution broadening. Using beamline P07 at PETRA III, Dippel et al. successfully measured PDFs from crystalline and amorphous thin films down to $3 \mathrm{~nm}$, 


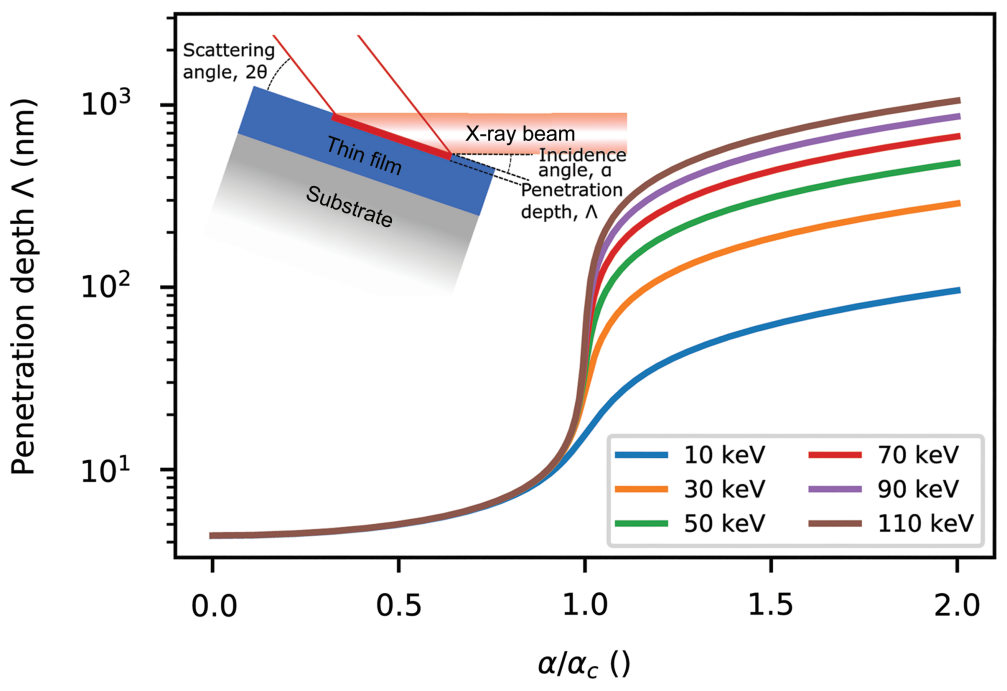

Figure 1

The penetration depth $\Lambda$ of X-rays in copper metal as a function of incidence angle $\alpha$ relative to the critical angle for total external reflection, $\alpha_{\mathrm{c}}$. Various energies are shown. For the grazing-incidence geometry (inset for reference) the penetration depth is small and energy independent for $\alpha<\alpha_{\mathrm{c}}$. This allows for selective enhancement of the thin film total scattering signal. Calculated based on equation (8) in the work of Feidenhans'l (1989).

with count times on the order of one second. This represents a factor of ten decrease in the minimum detectable film thickness, and a decrease of several orders of magnitude in the measurement time. Beyond this, their subsequent analysis results agree with previous studies on the same materials, demonstrating robustness and reproducibility.

Thin films are particularly relevant in technological applications including energy storage and conversion (Gao et al., 2017; Yang et al., 2017; Mao et al., 2017), catalysis (MoralesGuio et al., 2016; Ng et al., 2016; Xu et al., 2016), and electronics (Martin \& Rappe, 2017; Kelly et al., 2017; Deng et al., 2017). Dippel et al. have successfully demonstrated the feasibility of the GIPDF technique, bringing within reach a whole new class of in situ and operando structure studies on thin film devices and functional materials.

\section{Funding information}

This work was supported by US Department of Energy, Office of Science, Office of Basic Energy Sciences (DOE-BES) under contract No. DE-SC0012704.

\section{References}

Billinge, S. J. L., DiFrancesco, R. G., Kwei, G. H., Neumeier, J. J. \& Thompson, J. D. (1996). Phys. Rev. Lett. 77, 715-718.

Billinge, S. J. L. \& Kanatzidis, M. G. (2004). Chem. Commun. pp. 749 760.

Billinge, S. J. L., Turner, A. M. P., Thompson, J., Jeong, I.-K. \& Proffen, T. (2002). J. Appl. Cryst. 34, 536.

Chupas, P. J., Qiu, X., Hanson, J. C., Lee, P. L., Grey, C. P. \& Billinge, S. J. L. (2003). J. Appl. Cryst. 36, 1342-1347.

Deng, B., Tran, V., Xie, Y., Jiang, H., Li, C., Guo, Q., Wang, X., Tian, H., Koester, S. J., Wang, H., Cha, J. J., Xia, Q., Yang, L. \& Xia, F. (2017). Nat. Commun. 8, 14474.

Dippel, A.-C., Roelsgaard, M., Boettger, U., Schneller, T., Gutowski, O. \& Ruett, U. (2019). IUCrJ, 6, 290-298.

Egami, T. \& Billinge, S. J. L. (2012). Underneath the Bragg peaks: structural analysis of complex materials. Oxford: Elsevier.
Feidenhans'l, R. (1989). Surf. Sci. Rep. 10, 105-188.

Gao, P., Metz, P., Hey, T., Gong, Y., Liu, D., Edwards, D. D., Howe, J. Y., Huang, R. \& Misture, S. T. (2017). Nat. Commun. 8, 14559.

Guinier, A. (1994). X-ray diffraction in crystals, imperfect crystals, and amorphous bodies. New York: Dover.

Juhás, P., Davis, T., Farrow, C. L. \& Billinge, S. J. L. (2013). J. Appl. Cryst. 46, 560-566.

Keen, D. A. \& Goodwin, A. L. (2015). Nature, 521, 303-309.

Kelly, A. G., Hallam, T., Backes, C., Harvey, A., Esmaeily, A. S., Godwin, I., Coelho, J., Nicolosi, V., Lauth, J., Kulkarni, A., Kinge, S., Siebbeles, L. D. A., Duesberg, G. S. \& Coleman, J. N. (2017). Science, 356, 69-73.

Mao, Y., Li, G., Guo, Y., Li, Z., Liang, C., Peng, X. \& Lin, Z. (2017). Nat. Commun. 8, 14628.

Martin, L. W. \& Rappe, A. M. (2017). Nat. Rev. Mater. 2, 16087.

Masadeh, A. S., Božin, E. S., Farrow, C. L., Paglia, G., Juhas, P., Billinge, S. J. L., Karkamkar, A. \& Kanatzidis, M. G. (2007). Phys. Rev. B, 76, 115413.

Morales-Guio, C. G., Liardet, L. \& Hu, X. (2016). J. Am. Chem. Soc. 138, 8946-8957.

Ng, J. W. D., García-Melchor, M., Bajdich, M., Chakthranont, P., Kirk, C., Vojvodic, A. \& Jaramillo, T. F. (2016). Nat. Energ. 1, 16053.

Page, K., Hood, T. C., Proffen, Th. \& Neder, R. B. (2011). J. Appl. Cryst. 44, 327-336.

Peterson, P. F., Gutmann, M., Proffen, Th. \& Billinge, S. J. L. (2000). J. Appl. Cryst. 33, 1192.

Proffen, Th. \& Billinge, S. J. L. (1999). J. Appl. Cryst. 32, 572-575.

Proffen, Th. \& Neder, R. B. (1999). J. Appl. Cryst. 32, 838-839.

Qiu, X., Thompson, J. W. \& Billinge, S. J. L. (2004). J. Appl. Cryst. 37, 678.

Senn, M. S., Keen, D. A., Lucas, T. C. A., Hriljac, J. A. \& Goodwin, A. L. (2016). Phys. Rev. Lett. 116, 207602.

Tucker, M. G., Keen, D. A., Dove, M. T., Goodwin, A. L. \& Hui, Q. (2007). J. Phys. Condens. Matter, 19, 335218.

Vogt, T., Kanatzidis, M. G., Petkov, V., Trikalitis, P. N., Bozin, E. S. \& Billinge, S. J. L. (2002). J. Am. Chem. Soc. 124, 10157-10162.

Warren, B. E. (1969). X-ray diffraction. New York: Dover.

Xu, Z., Zhang, W., Weng, J., Huang, W., Tian, D. \& Huo, F. (2016). Nano Res. 9, 158-164.

Yang, C., Souchay, D., Kneiss, M., Bogner, M., Wei, H. M., Lorenz, M., Oeckler, O., Benstetter, G., Fu, Y. Q. \& Grundmann, M. (2017). Nat. Commun. 8, 16076. 